\title{
Análise clínica, cirúrgica e laboratorial de pacientes com conjuntivocálase
}

\author{
Clinical, surgical and laboratorial analysis of patients with conjunctivochalasis
}

\author{
Carlos Eduardo Borg'es Souza ${ }^{1}$ \\ Dinorah Piacentini Eng'el ${ }^{2}$ \\ Bruno Castelo Branco ${ }^{3}$ \\ Ana Luiza Höfling'-Lima ${ }^{4}$ \\ Luciene Barbosa de Souza ${ }^{5}$ \\ Jaison Barros ${ }^{6}$ \\ Denise de Freitas ${ }^{7}$
}

\section{RESUMO}

Objetivo: Avaliação clínica, cirúrgica e laboratorial de pacientes com conjuntivocálase. Métodos: Foi realizado exame oftalmológico antes e após tratamento cirúrgico em dez pacientes com conjuntivocálase avaliando os seguintes dados: acuidade visual, biomicroscopia do segmento anterior, padrão de coloração pela rosa bengala, teste de Schirmer e citologia de impressão. Resultados: Após a cirurgia todos os pacientes apresentaram melhora na sintomatologia e no padrão de rosa bengala. A citologia de impressão revelou metaplasia escamosa em oito pacientes. Conclusão: A cirurgia pode ser eficaz na melhora da sintomatologia dos pacientes com conjuntivocálase. Metaplasia escamosa foi achado freqüente nesses pacientes.

Descritores: Doenças da conjuntiva/cirurgia; Doenças da conjuntiva/terapia; Conjuntiva/ patologia; Síndromes do olho seco/patologia; Olho/patologia
Trabalho realizado no Departamento de Oftalmologia da Universidade Federal de São Paulo (UNIFESP).

${ }^{1}$ Médico pós-graduando do Departamento de Oftalmologia da Universidade Federal de São Paulo (UNIFESP)

${ }^{2}$ Médica residente do segundo ano do Departamento de Oftalmologia da Universidade Federal de São Paulo (UNIFESP).

3 Doutor em Oftalmologia pela Universidade Federal de São Paulo (UNIFESP).

${ }^{4}$ Chefe do Departamento de Oftalmologia da Universidade Federal de São Paulo (UNIFESP).

${ }^{5}$ Chefe do setor de Doenças Externas Oculares e Córnea do Departamento de Oftalmologia da Universidade Federal de São Paulo (UNIFESP).

${ }^{6}$ Tecnólogo do Departamento de Oftalmologia da Universidade Federal de São Paulo (UNIFESP).

Professora Livre Docente e afiliada do Departamento de Oftalmologia da Universidade Federal de São Paulo (UNIFESP).

Endereço para correspondência: Carlos Eduardo Borges Souza - Rua Três de Maio, 130 ap 174

São Paulo (SP) - CEP 04044-020

E-mail: ce.bsouza@uol.com.br

Recebido para publicação em 14.05.2003

Versão revisada recebida em 25.11.2003

Aprovação em 17.12.2003

\section{INTRODUÇÃ̃O}

Conjuntivocálase caracteriza-se pela presença de dobras redundantes, frouxas, não edematosas da conjuntiva bulbar inferior interposta entre o globo e a pálpebra inferior, como descrito inicialmente por Braunschweig em $1921^{(1)}$. Essa condição tende a ser bilateral, podendo localizar-se na parte lateral, central e medial da conjuntiva. É mais comumente encontrada em idosos, embora já tenha sido descrita em pacientes jovens com até quinze anos de idade ${ }^{(2)}$. Há associações com dermatocálase, lagoftalmo, pseudopterígio e pinguécula $a^{(3-4)}$.

Os sintomas são bastante variáveis, podendo não haver queixas nos casos leves, queixa de lacrimejamento e sensação de corpo estranho nos moderados e dor, irritação conjuntival, presença de hemorragia subconjuntival e úlcera marginal nos mais severos ${ }^{(5-8)}$.

Existem diferentes teorias sobre a fisiopatologia da conjuntivocálase ${ }^{(6,9)}$, assim como diferentes classificações; dentre estas se consideram: localização, número de dobras, altura das dobras em relação ao menisco lacrimal, mudança na altura das dobras em diferentes ducções e com pressão digital ${ }^{(5)}$.

O tratamento varia, de observação, nos casos leves a tratamento clínico, com lubrificantes sem preservativos associados a corticosteróides, antihistamínicos e oclusão noturna em casos moderados e severos, além de tratamento cirúrgico nos casos refratários ao tratamento clínico ${ }^{(5-8)}$. O tratamento cirúrgico inicialmente descrito por Braunscheiwg ${ }^{(1)}$ propõe incisão em forma de crescente na conjuntiva bulbar inferior a $5 \mathrm{~mm}$ do limbo seguido de fechamento primário com sutura. Outras técnicas se sucederam com modificações da técnica citada acima ${ }^{(5,7)}$ sendo que esta última utiliza a 
membrana amniótica para recobrir a área de retirada da conjuntiva.

O presente estudo pretende analisar dados clínicos, cirúrgicos e achados laboratoriais de pacientes com conjuntivocálase.

\section{MÉTODOS}

\section{1- Pacientes}

Foram estudados onze olhos de dez pacientes, examinados no ambulatório de Doenças Externas Oculares e Córnea do Departamento de Oftalmologia da Universidade Federal de São Paulo, com diagnóstico de conjuntivocálase, sem outra doença ocular ou sistêmica. Os pacientes tratados clinicamente com melhora dos sintomas e sinais foram excluídos do trabalho, permanecendo apenas os pacientes refratários ao tratamento clínico com indicação de cirurgia. Todos os pacientes incluídos nesse estudo assinaram consentimento livre e esclarecido aprovado pela comissão de ética e pesquisa da UNIFESP.

\section{2- Exame Oftalmológico}

Todos os pacientes foram submetidos a anamnese dirigida na qual respondiam questionário sobre a queixa principal que motivou a consulta e a presença de sintomas associados tais como lacrimejamento, sensação de corpo estranho, irritação, dor. Foi classificado como leve, moderado ou severo de acordo com a significância subjetiva dos sintomas pelo paciente.

Após esta, foi realizado a medida da acuidade visual, biomicroscopia à lâmpada de fenda, pressão intra-ocular, foto na lâmpada de fenda, oftalmoscopia indireta, teste de Schirmer sem anestésico, teste da coloração por rosa bengala a 1\%, e citologia de impressão da conjuntiva bulbar inferior (Quadro 1).

O teste de Schirmer tipo I (sem anestésico) avalia a secreção basal e reflexa da lágrima em resposta ao estímulo conjuntival. Foi colocado papel de filtro milimetrado na pálpebra inferior entre o terço medial e externo, o paciente permaneceu de olhos fechados, e após cinco minutos foi realizada a leitura. Valores menores que $10 \mathrm{~mm}$ foi considerado sugestivo de olho seco, superior a $15 \mathrm{~mm}$ como lacrimejamento em excesso.

\begin{tabular}{|c|c|c|c|c|c|c|c|}
\hline & AV & LF/Pio/FO & Foto & Sc total & RB & $\mathrm{Cl}$ & $\mathbf{R x}$ \\
\hline Pré & $x$ & $x$ & $x$ & $x$ & $x$ & $x$ & $x$ \\
\hline $1^{\circ} \mathrm{dia}$ & $(-)$ & $x$ & $(-)$ & $(-)$ & $(-)$ & $(-)$ & $(-)$ \\
\hline $7^{\circ} \mathrm{dia}$ & $x$ & $x$ & $x$ & $(-)$ & $(-)$ & $(-)$ & $(-)$ \\
\hline $2^{\circ} \mathrm{sem}$ & $x$ & $x$ & $X$ & $(-)$ & $(-)$ & $(-)$ & $(-)$ \\
\hline $3^{\circ} \mathrm{sem}$ & $x$ & $x$ & $x$ & $(-)$ & $(-)$ & $(-)$ & $(-)$ \\
\hline $4^{\circ} \mathrm{sem}$ & $X$ & $X$ & $x$ & $x$ & $x$ & $(-)$ & $(-)$ \\
\hline $2^{\circ}$ mês & $x$ & $x$ & $x$ & $x$ & $x$ & $(-)$ & $(-)$ \\
\hline
\end{tabular}

O teste da coloração por rosa bengala a 1\%, utiliza a classificação proposta por Van Bijsterveld que usa escala numérica que varia de zero a nove pontos, dividindo-se o olho em três áreas imagináveis: conjuntiva medial, córnea, e conjuntiva temporal. A intensidade de coloração varia de zero a três em cada área examinada. Pontuação igual ou maior que três em um olho é considerada anormal ${ }^{(10)}$.

\section{3- Técnica cirúrgica e tratamento pós-operatório:}

\subsection{Procedimento cirúrgico como descrito por Serrano e} Mora $^{(5)}$ :

- Peritomia inferior, de $90^{\circ}$, com tesoura de Wescott, duas incisões relaxantes radiais em direção ao fundo de saco inferior;

- A conjuntiva é separada da membrana de Tenon e elevada sobre a córnea. O tamanho da conjuntiva a ser ressecado é determinado pelo excesso de conjuntiva sobre a córnea, que passa pelo limbo inferior às seis horas;

- Refaz-se o fundo de saco inferior antes da ressecção da conjuntiva;

- Ressecção da conjuntiva previamente determinada;

- Sutura da conjuntiva ao limbo nas bordas usando nylon 10-0, ancorando na episclera e deixando uma margem de segurança de $1 \mathrm{~mm}$;

- Retirada da sutura dez a quinze dias após o procedimento.

3.2 Uso de colírio com a combinação de antibiótico e corticosteróide $\left(\right.$ Tobradex $^{\circledR}$ ), seis vezes por dia por sete dias, quatro vezes por dia até completar dez dias e depois mantido com o corticosteróide três vezes por dia até que todos os sinais inflamatórios desapareçam.

3.3 O tempo de seguimento dos pacientes foi de dois meses a contar da primeira consulta após a cirurgia.

\section{RESULTADOS}

A idade média dos pacientes foi de $72,5 \pm 5,0$ anos. Os sintomas e sinais mais freqüentes referidos pelos pacientes na anamnese dirigida em ordem decrescente foram:

- Sensação de corpo estranho 10 (100\%), epífora 8 (80\%), dor 6 (60\%), irritação ocular 3 (30\%), hemorragia subconjuntival 1(10\%) (Gráfico 1).

Nove dos dez pacientes referiram melhora dos sintomas após o tratamento cirúrgico, e apenas um paciente permane-

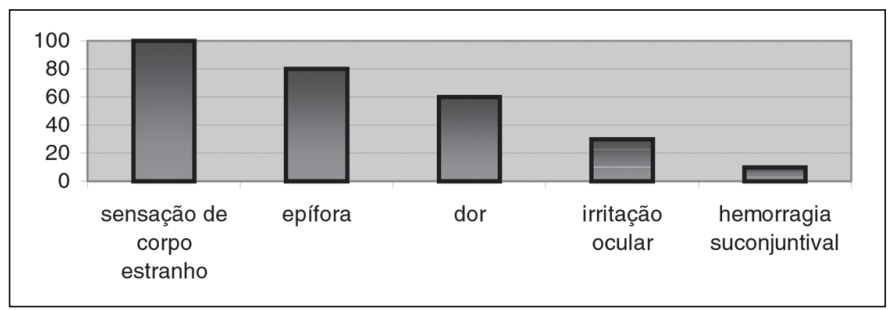

Gráfico 1 - Sinais e sintomas mais freqüentes apresentados em pacientes com conjuntivocálase 


\begin{tabular}{|c|c|c|c|c|c|c|c|c|}
\hline Pacientes & Sexo & Idade & Rosa & bengala & Schirmer & Citologia de impressão & Rosa bengala $\mathrm{PO}$ & Schirmer PO \\
\hline 1 & Fem & 75 a & & 6 & $20 \mathrm{~mm}$ & Grau II & 2 & $11 \mathrm{~mm}$ \\
\hline 2 & Masc & $67 \mathrm{a}$ & & 5 & $18 \mathrm{~mm}$ & Grau II & 2 & $18 \mathrm{~mm}$ \\
\hline 3 & Fem & $68 \mathrm{a}$ & & 7 & $15 \mathrm{~mm}$ & Grau II & 2 & $11 \mathrm{~mm}$ \\
\hline 4 & Fem & $68 \mathrm{a}$ & & 4 & $15 \mathrm{~mm}$ & Grau II & 0 & $10 \mathrm{~mm}$ \\
\hline 5 & Masc & $78 \mathrm{a}$ & & 5 & $15 \mathrm{~mm}$ & Grau I & 2 & $11 \mathrm{~mm}$ \\
\hline 6 & Fem & $66 \mathrm{a}$ & & 4 & $15 \mathrm{~mm}$ & Grau II & 2 & $10 \mathrm{~mm}$ \\
\hline 7 & Fem & $69 a$ & & 6 & $5 \mathrm{~mm}$ & Grau I & 0 & $12 \mathrm{~mm}$ \\
\hline 8 & Fem & $81 \mathrm{a}$ & & 5 & $5 \mathrm{~mm}$ & Grau II & 1 & $10 \mathrm{~mm}$ \\
\hline 9 & Fem & 76 a & & 4 & $15 \mathrm{~mm}$ & Grau II & 1 & $15 \mathrm{~mm}$ \\
\hline 10 & Masc & $74 a$ & & 8 & $27 \mathrm{~mm}$ & Grau III & 1 & $17 \mathrm{~mm}$ \\
\hline 11 & Fem & $75 a$ & & 4 & $5 \mathrm{~mm}$ & Grau II & 2 & $5 \mathrm{~mm}$ \\
\hline
\end{tabular}

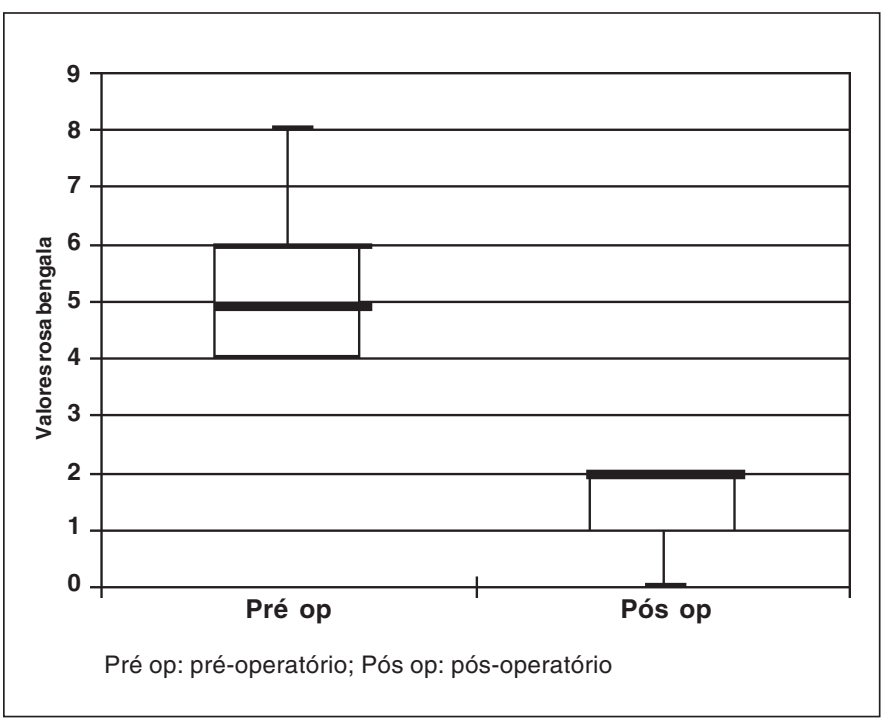

Gráfico 2 - Valores de rosa bengala dos pacientes com conjuntivocálase antes e após a cirurgia

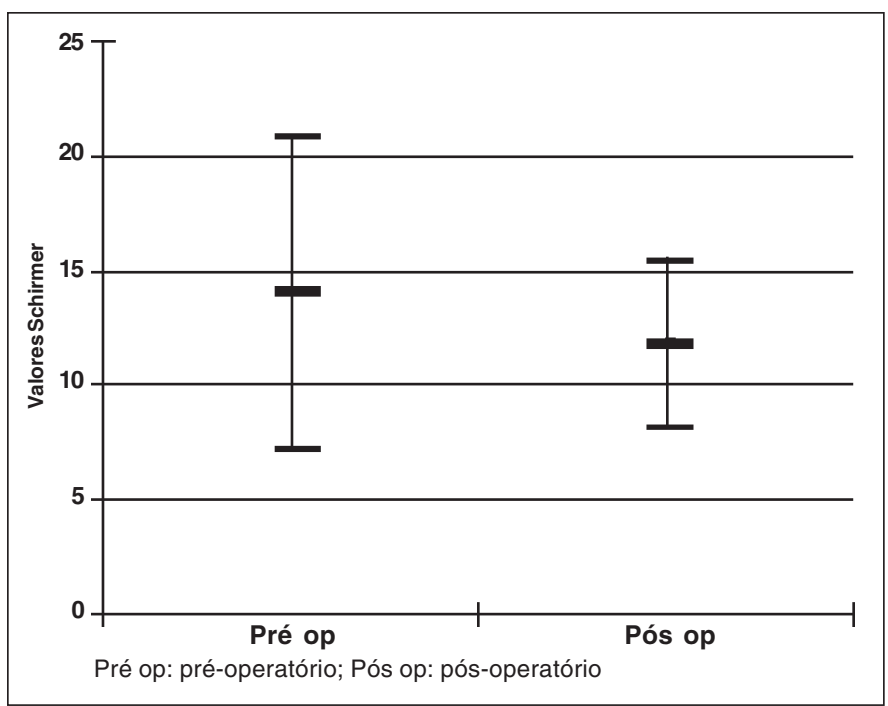

Gráfico 3 - Média dos valores do teste de Schirmer antes e depois da cirurgia em pacientes com conjuntivocálase, com respectivos valores de desvio padrão. A média pré-operatória $14,1( \pm 6,8)$; pós-operatória $11,8( \pm 3,7)$ ceu com queixa de epífora após a conclusão do estudo. Este paciente apresentava oclusão de ponto lacrimal para tratamento de olho seco associado.

Todos os pacientes apresentaram melhora no padrão da coloração por rosa bengala (Tabela 1 ). A mediana do padrão de rosa bengala pré-operatória foi de 5 , com valores de 4 e 6 para o percentil 25 e 75 respectivamente. A mediana pósoperatória foi de dois, com valores de 1 e 2 para o percentil 25 e 75 respectivamente (Gráfico 2).

Oito dos dez pacientes apresentaram citologia de impressão alterada com metaplasia escamosa grau 2 (de $0=$ bom a $3=$ ruim), e apenas dois pacientes obtiveram citologia de impressão dentro da normalidade (Grau 1). A mediana da citologia de impressão foi de dois (Tabela 1).

A média do teste de Schirmer pré-operatório foi de 14,1 $( \pm 6,8) \mathrm{mm}$, e a média do pós-operatório foi de $11,8( \pm 3,7) \mathrm{mm}$. (Gráfico 3).

\section{DISCUSS ÃO}

O presente estudo demonstrou ser fácil o diagnóstico clínico de conjuntivocálase, utilizando a propedêutica clínica habitual como já descrito por Braunschweig. Conjuntivocálase é achado comum, porém muitas vezes não diagnosticado ou tido como sendo alteração normal em indivíduos idosos (Figura 1).

A patogênese da conjuntivocálase ainda é incerta. É descrita a associação freqüente com pinguécula e pseudopterígio ${ }^{(4)}$. Postula-se que a conjuntivocálase seja causada por alteração elastótica da mesma forma que ocorre no pterígio ${ }^{(8,11)}$. Estudos demonstraram a presença exacerbada de metaloproteinases que podem degradar a matriz conjuntival e a Tenon, em fibroblastos cultivados depois de retirados da conjuntiva de pacientes com conjuntivocálase ${ }^{(12-13)}$.

Casos severos de conjuntivocálase podem ocasionar problemas relacionados à exposição, como lagoftalmo, dellen, e ulceração conjuntival ${ }^{(6-8)}$. A detecção de conjuntivocálase requer apenas métodos habituais de exame oftalmológico onde se percebe à biomicroscopia na lâmpada de fenda a presença de dobras redundantes não edematosas na conjuntiva bulbar 


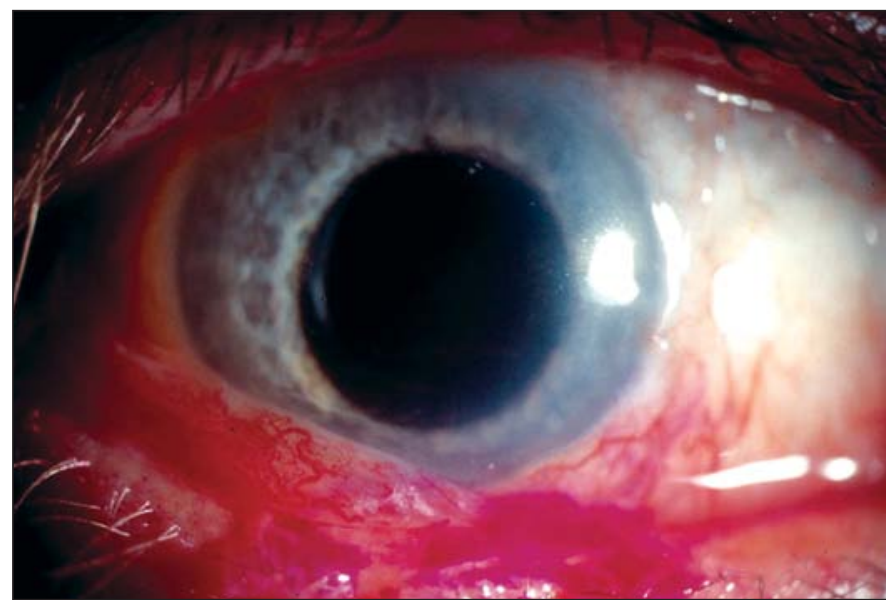

Figura 1 - Biomicroscopia do segmento externo ocular evidenciando a presença de conjuntivocálase. A conjuntiva encontra-se corada por rosa bengala a $1 \%$

inferior. A conjuntiva está muitas vezes associada à alteração no padrão da coloração da rosa bengala 1\% (Van Bijsterveld), e da fluoresceína com coloração da área não exposta da superfície ocular ${ }^{(3-4,6)}$. Em alguns casos, esses pacientes apresentam aumento da produção de lágrima, seja por lacrimejamento reflexo ou por alteração do escoamento da lágrima ${ }^{(6-7)}$. Estes achados podem auxiliar no diagnóstico de conjuntivocálase, uma vez que outras causas de lacrimejamento como obstrução das vias lacrimais, ectrópio, e entrópio estejam excluídas.

A citologia de impressão apresentou-se alterada na maioria dos casos severos de conjuntivocálase. Até onde nos foi possível averiguar na literatura nacional e internacional, este é o primeiro estudo a abordar este assunto. Nosso estudo constatou alteração na citologia de impressão da conjuntiva bulbar inferior em oito dos dez pacientes, o que pode sugerir alteração estrutural da conjuntiva no processo desencadeador da conjuntivocálase. Contudo, para melhor compreensão da patogênese da conjuntivocálase são necessários estudos mais aprofundados que permitam concluir se a alteração conjuntival é primária ou secundária a um processo de degeneração elastótica, desencadeado por reação inflamatória que degrada a matriz conjuntival.

Com a utilização do tratamento cirúrgico houve melhora na sintomatologia em nove dos dez pacientes. Todos os pacientes incluídos no trabalho foram anteriormente tratados clinicamente com lubrificantes sem preservativos, associados ou não a corticoesteróides tópicos sem melhora. $\mathrm{O}$ tratamento cirúrgico com a técnica de Serrano foi de fácil execução e levou a um resultado anatômico bom como anteriormente des- crito pelo próprio Serrano, sem retração do fundo de saco. E, principalmente, melhora clínica da sintomatologia como também observaram Daniel Muller, Renato Pires usando técnica com membrana amniótica ${ }^{(14)}$.

\section{ABSTRACT}

Purpose: To evaluate clinical, surgical and laboratorial findings in patients with conjunctivochalasis. Methods: Ophthalmologic examinations using $1 \%$ rose bengal, Schirmer test and impression cytology were performed in ten patients and after surgery. Results: Sintomatology improved in all patients on surgery. Impression cytology revealed metaplasia in eight patients. Conclusion: Surgical treatment may improve signs and symptoms in patients with conjuctivochalasis. Scamous metaplasia was a frequent finding in these patients.

Keywords: Conjunctival diseases/surgery; Conjunctival disease/therapy; Conjunctiva/pathology; Dry eye syndromes/ pathology; Eye/pathology

\section{REFERÊNCIAS}

1. Braunschweig P. Ueber faltenbildung der conjunctiva bulbi. Klin Monatsbl Augenheilkd. 1921;66:123-4.

2. Pinkerton OD. Bulbar conjunctivo-chalasis. Arch Ophthalmol. 1972;88(5):532

3. Wollenberg A. Pseudopterigium mit Faltenbildung der Conjunctiva bulbi. Klin Monatsbl Augenheilkd. 1922;68:221-4.

4. Li ZY, Wallace RN, Streeten BW, Kuntz BL, Dark AJ. Elastic fiber components and protease inhibitors in pinguecula. Invest Ophthalmol Vis Sci. 1991;32(5):1573-85.

5. Serrano F, Mora LM. Conjuntivochalasis: a surgical technique. Ophthalmic Surg 1989;20(12):883-4.

6. Hughes WL. Conjunctivochalasis. Am J Ophtalmol. 1942;25(1):48-51.

7. Meller D, Tseng SC. Conjunctivochalasis: literature review and possible pathophysiology. Surv Ophthalmol. 1998;43(3):225-32.

8. Jaros PA, DeLuise VP. Pingueculae and pterygia. Surv Ophthalmol. 1988;33 (1):41-9.

9. Jordan DR, Pelletier CR. Conjunctivochalasis. Can J Ophthalmol. 1996;31 (4):192-3.

10. van Bijsterveld OP. Diagnostic tests in the Sicca syndrome. Arch Ophthalmol. 1969;82(1):10-4.

11. Denti AV. Sulla formazione di una plica della congiuntiva bulbare. Boll Sper Med Chim. 1930;4(1):26-32.

12. Li DQ, Meller D, Liu Y, Tseng SC. Overexpression of MMP-1 and MMP -3 by cultured conjunctivochalasis fibroblasts. Invest Ophthalmol Vis Sci. 2000;41(2): 404-10.

13. Meller D, Li DQ, Tseng SC. Regulation of collagenase, stromelysin, and gelatinase $\mathrm{B}$ in human conjunctival and conjunctivochalasis fibroblasts by interleukin-1beta and tumor necrosis factor-alpha. Invest Ophthalmol Vis Sci. 2000;41(10):2922-9.

14. Pires RTF; Pires JLT; Tseng SCG. Transplante de membrana amniótica para reconstrução da superfície corneana e conjuntival. Arq Bras Oftalmol. 1999;62 (3):340-50. 